\title{
EFFECTS OF CHRONIC SUPPRESSION OF BIOELECTRIC ACTIVITY ON THE DEVELOPMENT OF SENSORY GANGLION EVOKED RESPONSES IN SPINAL CORD EXPLANTS
}

\author{
R. E. BAKER, ${ }^{1}$ M. A. CORNER, AND A. M. M. C. HABETS \\ Netherlands Institute for Brain Research, IJdijk 28, 1095 KJ Amsterdam, The Netherlands \\ Received June 20, 1983; Revised October 19, 1983; Accepted November 22, 1983
}

\begin{abstract}
Dorsal root ganglion (DRG) afferent terminals were identified, using electrophysiological techniques, within fetal mouse spinal cord cross-sections cultured in vitro. Afferent distribution patterns were monitored in explants grown for 3 to 6 weeks either in a serum-supplemented or in a serumfree, chemically defined medium (CDM). Bioelectrically active control explants from both series were compared with explants which had been reversibly silenced by chronic exposure to tetrodotoxin (TTX). The control (serum-grown) cultures showed a significant dorsal cord innervation preference, whereas in the corresponding TTX series there was an equal dorsoventral distribution. In the CDM series the mean number of DRG evoked responses was lower at first in TTX-grown than in control cultures, but with age in vitro there was a rise in excitability to normal levels. Spontaneous neuronal activity was abnormally low in cultures (serum as well as CDM-grown) which had been exposed to TTX. It is concluded that bioelectric activity may be an important factor in the proper regulation of synaptic connectivity and functional responsiveness in the developing spinal cord.
\end{abstract}

Sensory afferents from vertebrate dorsal root ganglia (DRG) enter and make a majority of their connections within the dorsal horn of the spinal cord (SC). This selectivity of connection occurs during development both in vivo (Gilbert and Stelzner, 1979) and, under the appropriate culturing conditions, in vitro (Crain, 1980; Crain and Peterson, 1981; Baker et al., 1982). "Spontaneous" neuronal discharges, i.e., the generation of action potentials in the absence of sensory input signals, develop during this same period in cord neurons as they mature in culture (Corner and Crain, 1972) as well as in situ (Provine and Rogers, 1977). Such activity could serve either as a source of "information" to the ingrowing afferent fibers regarding possible sites of interconnections, or as a "permissive" factor which is needed for the expression of interneuronal specific affinities (see Harris, 1981).

Action potential discharges appear to be required during early phases of development both for the survival (or growth) of certain types of neurons and for the formation of precise projection patterns between two normally interconnecting nerve centers. Thus, a significant decrease in the number of large spinal cord neurons occurs when cultured in the continued presence of tetrodotoxin (Bergey et al., 1981). Retinal ganglion cells are capable of

\footnotetext{
${ }^{1}$ To whom correspondence should be addressed.
}

selectively interconnecting with the appropriate visual centers in cat (Archer et al., 1982), goldfish (Meyer, 1982; Schmidt, 1982), and salamander (Harris, 1980) in the absence of afferent activity, but their terminal endings are relatively diffuse and abnormally shaped. Similarly, we have noted that fetal mouse DRG sensory afferents form preferential connections within the dorsal portion of serum-grown spinal cord explants even when cultured in the presence of the anesthetic xylocaine, but this dorsal preference was less pronounced than seen in controls (Baker et al., 1982). Since the number of cases was too small to verify this difference statistically, the first aim of the present investigation was to examine more closely the role of spontaneous bioelectric activity in the formation of selective DRG-SC connections.

Xylocaine-treated cultures differed from controls also in that, when tested in control medium, there was a reduction in the spread of DRG evoked discharges throughout the cord. Therefore, it is possible that endogenously generated neuronal firing is important for the full maturation of the network's electrophysiological response properties. Consistent with this idea is the observation that evoked activities in cord explants grown in chemically defined medium (CDM), where spontaneous action potentials are relatively abundant (Habets et al., 1981), become increasingly pronounced with age in vitro as compared with serum-grown explants (Baker et al., 
1982). Therefore, the second aim of the present study was to examine in greater detail the effect of chronic functional deprivation upon the development of spontaneous and of polysynaptic evoked activities within spinal cord explants. Tetrodotoxin (TTX) was chosen over xylocaine because of its more selective mode of action (reversible blockage of membrane sodium channels is the only known mechanism of TTX; Richie, 1979), and the experiments were carried out in serum-containing as well as in chemically defined nutrient media.

\section{Materials and Methods}

Cross-sections of 13- to 14-day fetal mouse spinal cord ( 0.5 to $1.0 \mathrm{~mm}$ thick), together with attached dorsal root ganglia, were plated onto collagen-coated plastic dishes (Nunc, $32 \mathrm{~mm}$ with air vents). Control cultures were grown in either a serum-supplemented (HSM) or a chemically defined medium. The former consisted of $100 \mathrm{ml}$ of Eagle's minimal essential medium (MEM) plus $20 \mathrm{ml}$ of horse serum, to which $10^{-5} \mathrm{M}$ cytosine arabinoside was added during the first week of incubation. The latter medium consisted of 1 part Dulbecco's modification of MEM, 1 part Ham's F-12, plus $10 \mu \mathrm{g} / \mathrm{ml}$ of insulin, 200 $\mu \mathrm{g} / \mathrm{ml}$ of transferrin, $40 \mathrm{nM}$ progesterone, $200 \mu \mathrm{M}$ putrescine, $60 \mathrm{nM}$ selenium $\left(\mathrm{Na}_{2} \mathrm{SeO}_{3}\right)$, and $0.1 \%$ albumin (Romijn et al., 1982). Experimental cultures differed from their respective controls only in that the growth medium also contained $10^{-6} \mathrm{M}$ TTX. The following series of cultures were run in parallel: HSM versus HSM-TTX, CDM versus CDM-TTX, and young versus old (CDMTTX). All cultures were refreshed once a week, during which time $0.01 \mathrm{ml}$ of freshly prepared $1 \%$ glutamine was added to the CDM and CDM-TTX cultures. Incubation temperature was maintained at $37^{\circ} \mathrm{C}$, while the $\mathrm{pH}$ was kept at 7.4 by a continuous gassing with $98 \%$ air and $2 \%$ $\mathrm{CO}_{2}$ (Auto $\mathrm{CO}_{2}$ Controller, Sirmac).

Cultures were selected for electrophysiological examination on the basis of their having visible fiber connections between the DRG and cord explant under phase contrast microscopy. The ages chosen for study were: 28 to 31 days in vitro for the HSM-TTX and CDM versus CDM-TTX cultures, and 21 versus 43 days in vitro for young and old CDM-TTX cultures, respectively. During the recording session the preparations were perfused with MEM while being constantly gassed with a mixture of air and $\mathrm{CO}_{2}$. Spontaneous and DRG evoked bioelectric activities were recorded at 16 evenly spaced points across the entire extent of the cord explant, using saline-filled glass micropipettes (tip diameters ranging from 5 to 15 $\mu \mathrm{m} ; 6$ to 10 megohms). The DRG were stimulated with similar micropipettes, using monopolar cathodal pulses (0.1 msec duration; up to $100 \mathrm{~V}$ amplitude). These electrodes were positioned under visual control with the aid of electronically driven micromanipulators. Explants from which no spontaneous and evoked activities could be recorded were excluded from the analysis.

The number and distribution of active recording sites (spontaneous and evoked) per culture were examined quantitatively, and the distribution of these sites was expressed as dorsal and ipsilateral percentages. For each experimental group the mean parameter-value and standard error were computed and subjected to the Student's $t$ test wherever appropriate (with one-tailed tests being used when the results were in the predicted direction). A "spreading factor"-defined as the total number of DRGpositive (all-latency) points divided by the number of fixed-latency points - was calculated for each preparation in which at least one such fixed-latency point was found (see Baker et al., 1982).

\section{Results}

Culture morphology and nerve fiber projection patterns. The overall morphology of the preparations did not differ in the present study from that reported previously ( $\mathrm{Ha}-$ bets et al., 1981; Baker et al., 1982). The HSM-grown cord explants flattened considerably during the first 7 to 10 days in vitro, losing much of their original cytoarchitectural detail. The TTX series of explants in general were thinner than the controls. CDM-grown cultures, on the other hand, did not flatten noticeably even after 6 weeks in vitro, retaining a recognizable spinal cord crosssectional appearance in almost all instances. In both media the DRG usually migrated away from the cord explant, giving rise to numerous fiber outgrowths. In most cases a number of these fibers became connected to the cord, either individually or by fiber bundles, often by way of the dorsal root remnant. In HSM the DRG tended to flatten into extensive monolayers, whereas in CDM they formed thick spheroid clusters.

Number and distribution of spontaneously active points. Spontaneous bioelectric activity was observed in all of the cultures examined, varying from continuous (often regular) to phasic firing patterns. The distribution of spontaneously active points in all groups showed no difference between dorsal or ventral cord regions (Figs. 1 and 2, \% dorsal). Nor was there a difference between ipsi- and contralateral cord projection patterns.

Cultures grown in HSM, when recorded at 4 weeks in vitro, yielded a greater number of active sites than did those grown in HSM-TTX (Fig. $1 ; p<0.005$ ). In the CDM series, in contrast, there was no significant difference at this age between control and experimental cultures with respect to the number of active points (Fig. 2). An age-related effect was noted in the CDM-TTX series, however, such that the oldest group showed significantly less spontaneous activity than either of the two younger groups ( $p<0.005$ for the 28 -day group, and $p<0.05$ for the 21-day group).

Number and distribution of all-latency evoked responses. Most of the activity recorded within the cord explants following DRG stimulation took the form of spike barrages with highly variable latencies for the majority of observed units. These active points were distributed about equally in all experimental series, with no significant preferences for dorsal versus ventral or ipsi- versus contralateral halves (Figs. 1 and 2, \%D).

The mean number of points showing evoked activity was the same in HSM and HSM-TTX cultures at 4 weeks in vitro (Fig. 1). The spreading factor, too, was comparable in both groups: $6.5( \pm 1.0, n=11)$ versus 5.2 ( $\pm 1.1, n=9$ ), respectively. In contrast, there was a significant difference between CDM and CDM-TTX cultures at that age (Fig. 2), with the treated group being less than half as responsive as the untreated group ( $p<$ 

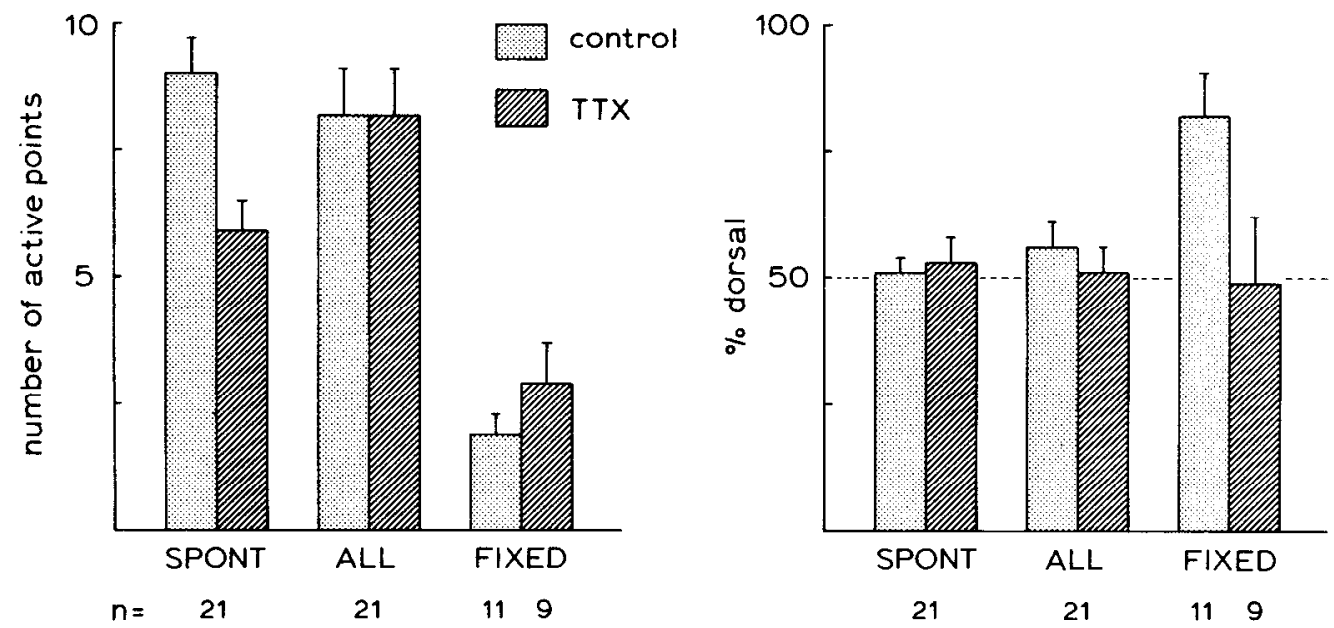

Figure 1. Bar graph representing the mean number (out of 16) and dorsal percentage ( \pm SEM) of spontaneous, all-latency $(A L L)$, and fixed-latency (FIXED) points in serum-grown cultures (HSM) at 28 to 31 days in vitro. The number of cultures examined is indicated by $n$.
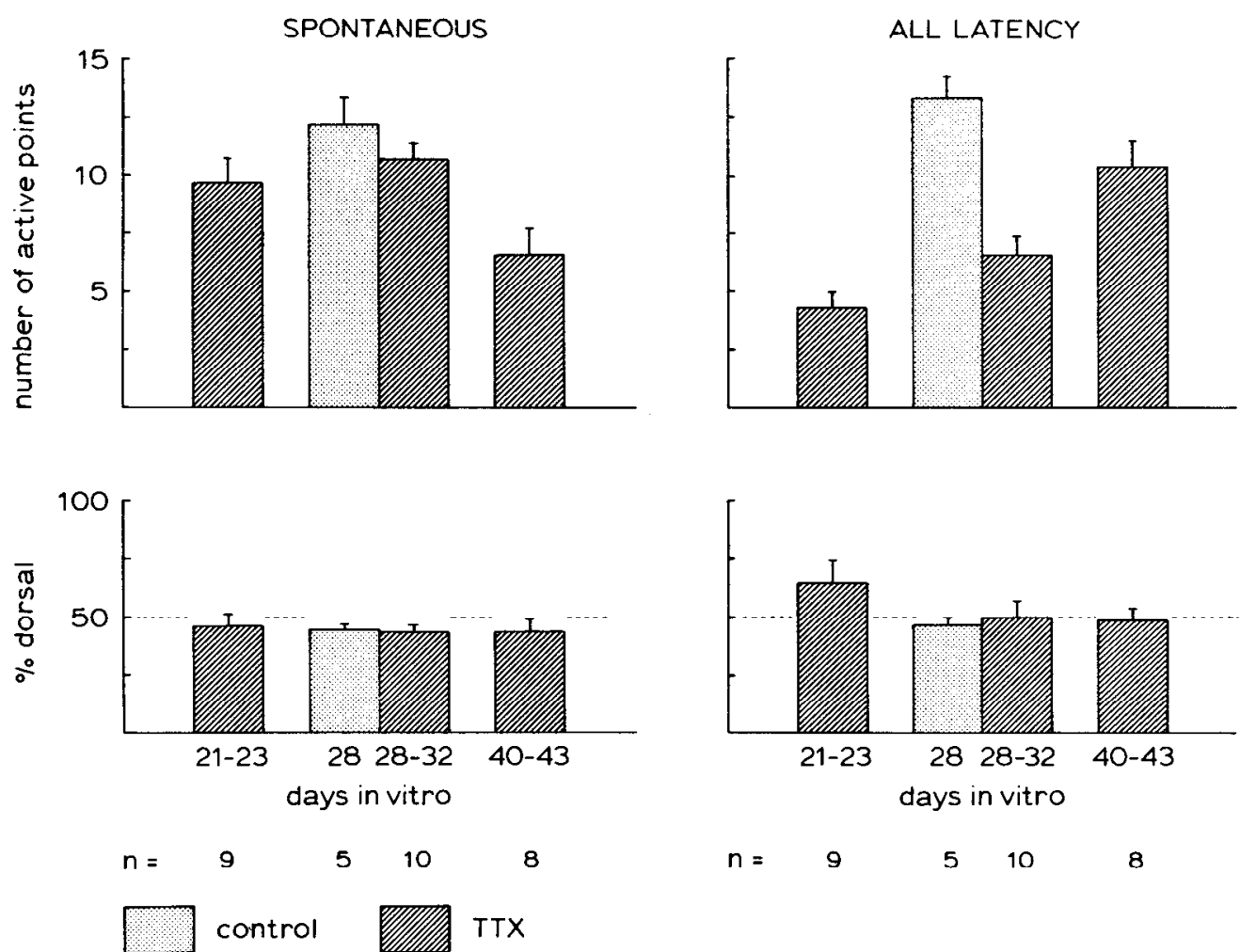

$\begin{array}{lllll}n= & 9 & 5 & 10 & 8\end{array}$

Figure 2. Bar graphs representing mean numbers (out of 16 , top row) and dorsal percentages (bottom row) of spontaneous and all-latency evoked responses in CDM-grown cultures $( \pm \mathrm{SEM})$. The number of cultures used is indicated by $n$.

$0.001)$. Also the spreading factor differed considerably: $2.8( \pm 0.6, n=6)$ for CDM-TTX versus $5.9( \pm 2.2, n=$ 4) for CDM controls. The number of all-latency evoked responses increased significantly with age in the TTXtreated (CDM) explants ( $p<0.01$ for the youngest versus the oldest group). The spreading factor showed a corresponding increase, from $4.3( \pm 1.3, n=5)$ in the youngest CDM-TTX cultures to $8.1( \pm 2.3, n=5)$ in the oldest ones.

Number and distribution of fixed-latency responses. As an indication of the presence of DRG-terminals, fixed- latency points were scored when one or more action potentials were present in the response to DRG stimulation which had a latency variation ("jitter") of $0.5 \mathrm{msec}$ or less (see Baker et al., 1982).

HSM control cultures showed a strong preference for DRG innervation of the dorsal rather than the ventral cord $(p<0.001)$. In HSM-TTX cultures, in contrast, the active points were about equally distributed between the dorsal and ventral halves (Fig. 1), even when DRG fiber entrances appeared to be predominantly dorsal (dorsal percentage $(\% \mathrm{D})=42 \pm 17, n=6)$. This differ- 
ence between the HSM and HSM-TTX groups is statistically significant $(p<0.025)$. In agreement with previous findings of a lack of dorsal specificity in DRG-cord explants cultured in CDM (Baker et al., 1982), the mean percentage of dorsal fixed-latency points in the CDMTTX series (21 to 43 days in vitro, $n=16$ ) was $\% \mathrm{D}=59$ \pm 11 ; for the 10 cultures which had a predominantly dorsal DRG fiber entrance, $\% \mathrm{D}=50 \pm 14$.

The youngest group of CDM-TTX explants showed a very strong ipsilateral cord innervation preference (ipsilateral percentage (\%I) $=90 \pm 10, p<0.001)$ which was not seen in the older CDM-TTX cultures $(\% \mathrm{I}=68 \pm$ 12). DRG fixed-latency responses were preferentially located on the ipsilateral side also in the HSM-TTX group (\% $=78 \pm 9, p<0.01$ ) but not in the HSM controls ( $\% \mathrm{I}=44 \pm 14)$.

The mean number of fixed-latency responses in HSMgrown controls was $1.9 \pm 0.4$ versus $2.9 \pm 0.8$ for HSMTTX cultures. For CDM-TTX cultures (all age groups combined) the corresponding value was $2.2 \pm 0.4$.

\section{Discussion}

Attempts at visualizing the patterns of fiber terminations within the spinal cord using histological staining techniques (e.g., cobalt, horseradish peroxidase, and degeneration) under the culturing conditions of the present study gave an unsatisfactory yield, although some successful cases have been reported previously (see Smalheiser et al., 1981; Baker et al., 1982). Thereforc, we were forced to rely upon the electrophysiological mapping of fixed-latency DRG evoked action potentials in the cord (see George and Marks, 1974; Baker et al., 1982).

It is unlikely that fixed-latency action potentials represent antidromically driven cord responses, on the basis of the following three arguments. First, cord cells can only rarely be backfilled with HRP administered to the DRG (Smalheiser et al., 1981; Baker et al., 1982). Second, pilot studies had shown that a period of several seconds was needed between test stimuli in order reproducibly to evoke fixed-latency responses in cord explants. Finally, if the fixed-latency activity were substantially contaminated by antidromically driven cord cells, this would imply that there is a selective localization of such cells in the dorsal half of the cord (observed dorsal preference of connections in HSM). Their existence should then also give a similar dorsal preponderance of SC-DRG connections in CDM, which is not the case (Baker et al., 1982 and present study). Nor is there any reason to expect that the addition of the simple hexose sugar galactose to CDM, which restores the dorsal innervation preferences (Baker et al., 1983), would save such hypothetical cells from extinction.

The present study reveals that, following chronic suppression of spontaneous bioelectric activity by means of TTX, the distribution pattern of sensory afferents within mouse spinal cord explants (if grown in the presence of horse serum) differs significantly from controls. The apparent lack of specificity in HSM-TTX cultures is surprising in view of our previous results in serumgrown cultures chronically silenced by xylocaine, where the fixed-latency responses did show a significant dorsal cord preference (Baker et al., 1982) which was, however, less pronounced than in the controls. Some degree of innervation specificity, even in the absence of functional activity, would be expected on the basis of the findings in visual systems. In fish, blocking retinal bioelectric activity by means of TTX leads to the formation of topographically well organized but relatively diffuse visual projections (see Meyer, 1982; Schmidt, 1982). Similarly, neural activity is required for the development and maintenance of the adult 1:1 ratio of motoneuron-muscle fiber innervation in mammalian skeletal muscles (see Jansen et al., 1973; Thompson et al., 1979). Thus, in several systems, bioelectric activity appears to be involved in the "fine tuning" of synaptic connectivity (see Harris, 1981). However, all of the above examples report on the effects of suppressing presynaptic activity (which corresponds to the situation existing in our control preparations, DRG's not being spontaneously active in vitro). In our experimental groups, also the postsynaptic bioelectric responses were eliminated (by TTX treatment in the present study, and by xylocaine in the study of Baker et al., 1982). Therefore, the present results suggest that postsynaptic activity may be equally important for the development of selective interneuronal connections (see also Freeman, 1977; Schmidt, 1982).

Several mechanisms may underlie such developmental regulation in the neuron: $(a)$ changes in the production of trophic factors (e.g., Freeman, 1977; Walicke et al., 1977; Betz et al., 1980); (b) inability of the silenced neurons to compete successfully with active neighbors for target sites (e.g., Mark, 1980; Purves and Lichtman, 1980; Sargent and Dennis, 1981); and (c) interference with the metabolism of vital cellular components necessary for proper maturation. By silencing bioelectric activity, TTX interferes with a variety of metabolic processes, including a significant decrease in enzyme systems responsible for electron transport and oxidative phosphorylation (Wong-Riley et al., 1978; Wong-Riley and Riley, 1983) and in choline acetyltransferase activity (Bergey et al., 1981; Jackson et al., 1982). One result of such decreases would be a lowered level of synthesis of many types of membrane-associated macromolecules, some of which are presumably important in the construction of nerve cell membranes and intercellular-specific recognition sites.

The extent of polysynaptic (i.e., all-latency) evoked DRG discharges in the CDM series of experiments suggests that spontaneous action potentials, in addition to affecting synaptic connectivity, can influence the responsiveness of developing spinal cord networks to incoming stimuli. Thus, the "spreading factor" for evoked responses was much higher in control cultures at 28 days in vitro than in those treated with TTX. It is tempting to attribute this decreased network excitability to the loss of certain cell types (Bergey et al., 1981). However, network excitability is only retarded in the continued absence of bioelectric activity, and by 6 weeks in vitro the number of all-latency evoked discharges had become comparable to that found in untreated CDM-grown preparations (also see Baker et al., 1982). The importance of bioelectric activity for neural development also in serum- 
containing medium is suggested by recent electron microscopic studies showing an early retardation of synaptic growth in TTX-treated cerebral cortex cultures (Janka and Jones, 1982; Van Huizen et al., 1982).

Spontaneous bioelectric activity, too, becomes reduced in amount as a result of chronic blockade of action potentials during the maturation of spinal cord tissues in vitro, at lcast in the HSM series. Furthermore, TTXtreated explants in the CDM series showed a drop in spontaneous activity in the oldest age group, instead of the continued increase which is characteristic of untreated cultures grown in this medium (Baker et al., 1982). The lack of correspondence between TTX effects on spontaneous and (all-latency) evoked activities, respectively, suggests that the reduction in the former cannot be explained simply on the basis of lower overall excitability levels within the network. Presumably, the maturation of those neurons which are ultimately responsible for endogenous discharges in these cord cultures (Corner and Crain, 1972) depends upon the presence of bioelectric activity in order for full maturation to occur. The phenomenon of "kindling" (see Racine, 1978), as well as the reported effectiveness of chronic electrical stimulation in altering both structure and function in isolated adult cortex neurons in situ (e.g., Rutledge, 1969), suggests that neurophysiological activity remains important throughout life.

\section{References}

Archer, S. M., M. W. Dubin, and L. A. Stark (1982) Abnormal development of kitten retino-geniculate connectivity in the absence of action potentials. Science 217: 743-745.

Baker, R. E., A. M. M. C. Habets, E. Brenner, and M. A. Corner (1982) Influence of growth medium, age in vitro and spontancous bioclectric activity on the distribution of sensory ganglion-evoked activity in spinal cord explants. Dev. Brain Res. 5: 329-341.

Baker, R. E., M. A. Corner, and M. Kleiss (1983) Effect of charged membrane-associated molecules on the formation of selective innervation patterns in fetal mouse spinal corddorsal root ganglion cultures. Neurosci. Lett. 41: 321-324.

Bergey, G. K., S. C. Fitzgerald, B. K. Schrier, and P. G. Nelson (1981) Neuronal maturation in mammalian cell cultures is dependent on spontaneous bioelectric activity. Brain Res. 207: 49-58.

Betz, W. J., J. H. Caldwell, and R. R. Ritchester (1980) Sprouting of active nerve terminals in partially inactive muscles of the rat. J. Physiol. (Lond.) 303: 281-297.

Corner, M. A., and S. M. Crain (1972) Patterns of spontaneous hioelectric activity during maturation in culture of fetal rodent medulla and spinal cord tissues. J. Neurobiol. 2: 2545.

Crain, S. M. (1980) Development of specific sensory-evoked synaptic networks in organized CNS tissue culture. In Tissue Culture in Neurobiology, E. Giacobini, A. Vernadakis, and A. Shahar, eds., pp. 169-185, Raven Press, New York.

Crain, S. M., and E. R. Peterson (1981) Selective innervation of target regions within fetal mouse spinal cord and medulla explants by isolated dorsal root ganglion in organotypic cocultures. Dev. Brain Res. 254: 341-362.

Freeman, J. A. (1977) Possible regulatory function of acetylcholine receptor in maintenance of retinotectal synapses. Nature 269: 218-222.
George, S. A., and W. B. Marks (1974) Optic nerve terminals in the frog: Shape and orientation inferred from electrophysiological measurements. Exp. Neurol. 42: 467-482.

Gilbert, M., and D. J. Stelzner (1979) The development of descending and dorsal root connections in the lumbosacral spinal cord of the postnatal rat. J. Comp. Neurol. 184: 821838.

Habets, A. M. M. C., R. E. Baker, E. Brenner, and M. A. Corner (1981) Chemically defined medium enhances bioelectric activity in mouse spinal cord-dorsal root ganglion cultures. Neurosci. Lett. 22: 51-56.

Harris, W. A. (1980) The effects of eliminating impulse activity on the development of the retinotectal projection in salamanders. J. Comp. Neurol. 194: 303-317.

Harris, W. A. (1981) Neural activity and development. Annu. Rev. Physiol. 43: 689-710.

Jackson, M. B., H. Lecar, D. E. Brenneman, S. Fitzgerald, and P. G. Nelson (1982) Electrical development in spinal cord cell culture. J. Neurosci. 2: 1052-1061.

Janka, Z., and D. G. Jones (1982) Junctions in rat neocortical explants cultured in TTX-, GABA-, and $\mathrm{Mg}^{++}$-environments. Brain Res. Bull. 8: 273-278.

Jansen, J. K. S., T. Lømo, K. Nicolaysen, and R. H. Westgaard (1973) Hyperinnervation of skeletal muscle fibers: Dependence on muscle activity. Science 181: 559-561.

Mark, R. F. (1980) Synaptic repression at neuromuscular junction. Physiol. Rev. 60: 355-395.

Meyer, R. L. (1982) Tetrodotoxin blocks the formation of ocular dominance columns in goldfish. Science 218: 589-591.

Provine, R. R., and L. Rogers (1977) Development of spinal cord bioelectric activity in spinal chick embryos and its behavioral implications. J. Neurobiol. 8: 217-228.

Purves, D., and J. W. Lichtman (1980) Elimination of synapses in the developing nervous system. Science 210: 153157.

Racine, R. (1978) Kindling: The first decade. Neurosurgery 3: 234-252.

Richie, J. M. (1979) A pharmacological approach to sodium channels in myelinated axons. Annu. Rev. Neurosci. 2: $341-$ 362.

Romijn, H. J., A. M. M. C. Habets, M. T. Mud, and P. S. Wolters (1982) Nerve outgrowth, synaptogenesis and bioelectric activity in fetal rat cerebral cortex tissue cultured in serum-free, chemically defined medium. Dev. Brain Res. 2: $583-589$.

Rutledge, L. T. (1969) Effect of stimulation on isolated cortex. In Basic Mechanisms of the Epilepsies, H. H. Jasper, A. A. Ward, and A. Pope, eds., pp. 349-355, Little, Brown, Boston.

Sargent, P. B., and M. J. Dennis (1981) The influence of normal innervation upon abnormal synaptic connections between frog parasynaptic neurons. Dev. Biol. 81: 65-73.

Schmidt, J. T. (1982) The formation of retinotectal projections. Trends Neurosci. 6: 111-115.

Smalheiser, N. R., E. R. Peterson, and S. M. Crain (1981) Specific neuritic pathways and arborizations formed by fetal mouse dorsal root ganglion cells within organized spinal cord explants in culture: A peroxidase-labeling study. Dev. Brain Res. 2: 383-396.

Thompson, W., P. D. Kuffler, and J. K. S. Jansen (1979) The effect of prolonged, reversible block of nerve impulses on the elimination of polyneuronal innervation of new-born rat skeletal muscle fibers. Neuroscience $4: 271-281$.

Van Huizen, F., H. J. Romijn, P. S. Wolters, and A. M. M. C. Habets (1982) Development of synaptic contacts in fetal rat cerebral cortex cultures in relation to developing bioelectric activity. Neurosci. Lett. Suppl. 10: 499.

Walicke, P. A., R. B. Campenot, and P. H. Patterson (1977) Determination of transmitter function by ncuronal activity. 
Proc. Natl. Acad. Sci. U. S. A. 74: 5767-5771.

Wong-Riley, M., and D. A. Riley (1983) The effect of impulse blockage on cytochrome oxidase activity in the cat visual system. Brain Res. 261: 185-193.
Wong-Riley, M., M. M. Merzenich, and P. A. Leake (1978) Changes in endogenous enzymatic reactivity to $\mathrm{DAB}$ induced neuronal inactivity. Brain Res. 141: 185-192. 